\title{
Memory is preserved in older adults taking AT1 receptor blockers
}

\author{
Jean K. Ho ${ }^{*}$, Daniel A. Nation ${ }^{*}$ for the Alzheimer's Disease Neuroimaging Initiative
}

\begin{abstract}
Background: Prior work suggests that some but not all antihypertensive treatments may benefit cognition and risk for Alzheimer's disease, independent of stroke. Angiotensin II receptor blockers (ARBs) have been highlighted as one antihypertensive drug class that may confer greatest benefit.

Methods: The participants comprised 1626 nondemented adults, aged 55-91 years, recruited from Alzheimer's Disease Neuroimaging Initiative sites. Three groups were compared: ARB users (HTN-ARBs), other antihypertensive drug users (HTN-Other), and normotensives. In post hoc analyses, we also examined (1) users of ARBs and angiotensin-converting enzyme inhibitors (ACEIs), (2) users of blood-brain barrier (BBB)-crossing ARBs and users of non-BBB-crossing ARBs, and (3) users of BBB-crossing ARBs and ACEls (BBB crossers) and users of non-BBB-crossing ARBs and ACEls (BBB noncrossers). Groups were compared regarding cognition and magnetic resonance imaging measures of brain volume and white matter hyperintensities (WMH), using analysis of covariance and multilevel models.
\end{abstract}

Results: At baseline, the HTN-Other group performed worse than normotensives on Rey Auditory Verbal Learning Test (RAVLT) Immediate Recall ( $p=0.002)$, Delayed Recall $(p<0.001)$, Recognition Memory $(p=0.001)$, and Trails A $(p<0.001)$ and B $(p=0.01)$. ARB users performed better than the HTN-Other group on Recognition Memory $(p=0.04)$ and worse than normotensives on Trails A $(p=0.04)$. The HTN-Other group performed worse than normotensives on Logical Memory Immediate $(p=0.02)$ and Delayed Recall over the 3-year follow-up $(p=0.007)$. Over the follow-up period, those taking BBB-crossing ARBs performed better than the HTN-Other group on AVLT Delayed Recall $(p=0.04)$, Logical Memory Immediate $(p=0.02)$, and Delayed Recall $(p=0.05)$. They also had fewer WMH than the HTN-Other group $(p=0.008)$ and those taking non-BBB-crossing ARBs $(p=0.05)$. There were no group differences in brain volume. Users of BBB-crossing medications (ARBs or ACEIs) showed better performance on AVLT Delayed Recall over time than all other groups, including normotensives (all $p<0.01$ ), and had less WMH volume over time than the BBB noncrossers group $(p=0.03)$, although they had more WMH volume than normotensives $(p=0.01)$. The BBB noncrossers group performed worse than normotensives on Logical Memory Delayed Recall over time $(p=0.01)$, but the BBB crossers group was not significantly different $(p=0.13)$.

Conclusions: Hypertensive participants demonstrated worse baseline memory and executive function, as well as greater memory decline, over the 3-year follow-up than normotensives, unless they were ARB users, who showed preserved memory compared with those taking other antihypertensive drugs. Users of BBB-crossing ARBs showed superior memory performance over time compared with other antihypertensive drug users and had less WMH volume. Users of BBB-crossing medications (ARBs or ACEIs) showed better list-learning memory performance over time than all other groups, including normotensives, and had less WMH volume over time than users of non-BBB-crossing medications. These findings demonstrate that ARBs, especially those of the BBB-crossing variety, are associated with greater memory preservation and less WMH volume than other antihypertensive medications.

Keywords: Blood pressure, Antihypertensive medication, Angiotensin receptor blockers, Memory, Alzheimer's disease

* Correspondence: jeanho@usc.edu; danation@usc.edu

Department of Psychology, University of Southern California, 3620 South

McClintock Avenue, SGM 1010, Los Angeles, CA 90089-1061, USA

(c) The Author(s). 2017 Open Access This article is distributed under the terms of the Creative Commons Attribution 4.0 International License (http://creativecommons.org/licenses/by/4.0/, which permits unrestricted use, distribution, and reproduction in any medium, provided you give appropriate credit to the original author(s) and the source, provide a link to the Creative Commons license, and indicate if changes were made. The Creative Commons Public Domain Dedication waiver (http://creativecommons.org/publicdomain/zero/1.0/) applies to the data made available in this article, unless otherwise stated. 


\section{Background}

Hypertension is the most common condition observed in primary care and among the most treatable risk factors for illness [1]. Hypertension is also an established risk factor for cognitive decline, Alzheimer's disease, and vascular dementia [2]. These and other findings support the vascular hypothesis of Alzheimer's disease, which postulates that cerebrovascular disease and dysfunction, due to hypertension and other vascular risk factors, contribute to this dementing illness through effects on cerebral perfusion and blood-brain barrier (BBB) compromise [3, 4]. Therefore, work surrounding antihypertensive treatments and their possible salutary effects on preserving cognition through improved vascular health forms a promising area of research [5].

Hypertension and vascular risk factors have been linked to decreased cognitive function [6] beyond expected declines in processing speed and memory associated with normal aging [7]. For example, hypertension and diabetes mellitus have been found to be positively associated with cognitive decline in middle-aged adults, a decline that is greater than that experienced by healthier counterparts without these risk factors [8]. Midlife hypertension and elevations in serum cholesterol levels have also been linked to the development of mild cognitive impairment (MCI) in later life [9]. Relative to normotensive individuals, hypertensive persons exhibit worse performance in many domains of cognitive function, including episodic memory [10], working memory and executive function [11], attention and psychomotor speed [12], and language [13]. These cognitive deficits may be underpinned by hypertensionrelated vascular brain injury caused by alterations in cerebral hemodynamics and cerebral small vessel disease [14]. Functional implications of these vascular changes have been demonstrated in mouse models, where arterial hypertension has been found to increase the permeability and dysfunction of the BBB as well as to reduce cerebral blood flow [15]. Increased blood pressure, particularly pulse pressure, has also been linked to amyloid- $\beta$ (A $\beta)$ deposition [16, 17], tau-mediated neurodegeneration [18], and Alzheimer's disease [19].

Despite these clear links between blood pressure and both dementia and Alzheimer's disease, studies investigating cognitive benefits of blood pressure control have been mixed. Whereas some randomized controlled trials (RCTs)-Medical Research Council Elderly (MRC-Elderly), Systolic Hypertension in the Elderly Program (SHEP), Perindopril Protection against Recurrent Stroke Study (PROGRESS), Systolic Hypertension in Europe (SYSTEUR) trial, and the observational SYST-EUR 2 study-indicated some benefit, the Hypertension in the Very Elderly Trial assessing cognitive decline and dementia incidence (HYVET-COG), the Telmisartan Randomised AssessmeNt Study in ACE iNtolerant subjects with cardiovascular Disease (TRANSCEND), and the Ongoing Telmisartan Alone and in Combination with Ramipril Global Endpoint Trial (ONTARGET) as well as a Cochrane review did not $[20,21]$. Of the former, it must be noted that the MRC-Elderly study [22] was not a double-blind trial and included only two longitudinal cognitive outcome measures. The PROGRESS study did not find a clear effect of hypertensive treatment on dementia, but the researchers did report that treatment reduced the risk of cognitive decline by approximately $20 \%$ and reduced the risk of "cognitive decline with recurrent stroke" by approximately 50\% [23]. The study authors suggested that benefits of hypertensive treatment in their sample may have been the result of stroke prevention as opposed to direct influence on dementia or cognitive impairment [23]. In the SHEP study [24], a double-blind RCT, researchers found slight positive effects of treatment on cognitive, physical, and leisure functioning but failed to find any significant difference in the incidence of cognitive deterioration between participants on active hypertension treatment and placebo. However, a report on the reanalysis of these data indicated that differential dropout from the treatment and placebo groups may have biased these results toward a null effect [25].

In studies suggesting possible protective effects, individuals treated for hypertension have been found to have less Alzheimer's disease neuropathology than both untreated hypertensive individuals and normotensive persons, evidenced in lower neuritic plaque counts and fewer neurofibrillary tangles of hyperphosphorylated tau protein [26]. Compared with individuals who have never taken any antihypertensive drugs, individuals treated with these medications exhibit decreased risk of all-cause dementia, with an $8 \%$ risk reduction for every year of use in individuals $<75$ years old, with similar estimates for Alzheimer's disease [27]. Authors of a recent meta-analysis of 19 randomized trials and 11 studies examining the relationships among antihypertensive drug use, cognition, and dementia incidence provided support for the perspective that antihypertensive treatment may have beneficial effects on cognition [28]. Of the many antihypertensive drug classes available, angiotensin II receptor blockers (ARBs) were highlighted as the one class that may confer the greatest benefit [28].

Therefore, in the present study, we sought to investigate whether use of ARBs would confer protective effects on cognition greater than those of other antihypertensive drugs. Our specific hypotheses were that (1) normotensive individuals would show the best preservation of neuropsychological function, followed by ARB users, who in turn would outperform users of other antihypertensive drugs; (2) ARB users would show better memory and executive function than users of other antihypertensive drugs, in line 
with positive effects reported elsewhere [29, 30]; and (3) in comparison with users of other antihypertensive drugs, ARB users would show attenuation of cognitive decline over time.

\section{Methods}

Data were obtained from the Alzheimer's Disease Neuroimaging Initiative (ADNI) database (adni.loni.usc.edu). The primary goal of ADNI is to test whether neuroimaging, other biological markers, and clinical and neuropsychological assessments can be combined to measure the progression of MCI and early Alzheimer's disease. ADNI is the result of efforts of many coinvestigators from a range of academic institutions and private corporations, and subjects have been recruited from more than 50 sites across the United States and Canada. Participants are recruited via newsletters, Internet-based communications, direct mail, and press releases. Inclusion criteria include aged 55-91 years, permitted medications stable for 4 weeks, study partner who can accompany participant to visits, Geriatric Depression Scale score less than 6, Hachinski Ischemic Scale score less than or equal to 4 , adequate visual and auditory acuity, good general health, 6 grades of education or work history equivalent, and ability to speak English or Spanish fluently. Exclusion criteria for cognitively normal subjects and participants with $\mathrm{MCI}$ include any significant neurological disease or history of significant head trauma. For more information, see www.adni-info.org.

\section{Participants}

The participants comprised 1626 nondemented ADNI-1, ADNI-Grand Opportunity, and ADNI-2 participants who were classified as either cognitively normal or having $\mathrm{MCI}$ at screening evaluation. Criteria for MCI were (1) subjective memory complaint reported by the participant or informant, (2) Mini Mental State Examination (MMSE) scores between 24 and 30 (inclusive), (3) global Clinical Dementia Rating of 0.5, (4) scoring below educationadjusted cutoffs for delayed free recall on Story A of the Wechsler Memory Scale-Revised (WMS-R) Logical Memory II subtest, and (5) general cognition and functional performance preserved to the extent that one would not qualify for a diagnosis of Alzheimer's disease [31].

\section{Blood pressure medication groups}

Eight hundred four participants (49.4\% of the total sample) reported using at least one of eight classes of antihypertensive drugs: ARBs, angiotensin-converting enzyme inhibitors (ACEIs), $\beta$-blockers, calcium channel blockers, $\alpha_{1}$-adrenergic blockers, $\alpha_{2}$-agonists or other centrally acting drugs, diuretics, or direct vasodilators. Of these, 183 (11.2\% of the total sample) were taking ARBs and were classified as ARB users (HTN-ARBs). Six hundred twenty-one (38.3\% of the total sample) were taking other antihypertensive medications and were classified as users of other antihypertensive drugs (HTN-Other). The remaining 822 ( $50.5 \%$ of the total sample) had no documented history of hypertension and were classified as normotensive. To ensure that untreated hypertensive subjects were excluded, blood pressure measures were used to exclude those with values at or above stage 2 hypertension $(160 / 100 \mathrm{mmHg} ; n=40)$. We did not use the more conservative cutoffs recommended for treatment of older adults $(150 / 90 \mathrm{mmHg})$, owing to the unreliability of a single blood pressure measure and the likelihood that the research environment may have artificially produced transient, mild elevations in blood pressure in normotensive individuals. Thus, the final sample consisted of 1586 participants (see Additional file 3).

Given that ACEIs also act on the renin-angiotensinaldosterone system (RAAS), and given that the capability of medications to cross the BBB is related to drug efficacy, in post hoc analyses we also examined (1) users of ARBs and ACEIs $(n=464)$; (2) users of BBB-crossing ARBs of valsartan, telmisartan, and candesartan $(n=72$ ARB crossers) and users of non-BBB-crossing ARBs of irbesartan, olmesartan, losartan, and eprosartan [32] ( $n=102$ ARB noncrossers); and (3) users of BBB-crossing ARBs and BBB-crossing ACEIs of captopril, fosinopril, lisinopril, perindopril, trandolapril, or zofenopril $(n=277 \mathrm{BBB}$ crossers) and users of non-BBB-crossing ARBs and non-BBB-crossing ACEIs of benazepril, enalapril, moexepril, quinapril, and ramipril [33] ( $n=187$ BBB-noncrossers).

\section{Physiological, clinical, and genetic data}

Physiological measures included seated brachial artery systolic and diastolic blood pressures. Pulse pressure was computed as systolic minus diastolic pressure. Data on blood pressure indices were available for $98.8 \%$ of the sample. Body mass index (BMI) was calculated as weight (in kilograms) divided by height (in meters) squared. Data on BMI were available for $98.8 \%$ of the sample. Blood samples were used to determine apolipoprotein $\mathrm{E}$ (APOE) $\varepsilon 4$ allele carrier status. Participants were categorized as those with or without one or more copies of the APOE $\varepsilon 4$ allele. APOE genotype data were available for $85.3 \%$ of the sample.

\section{Vascular risk factors}

Vascular risk factor burden was assessed on the basis of medical history and physical examination at baseline and screening using criteria adapted from the Framingham Stroke Risk Profile [34] and Framingham Coronary Risk Profile [35]. Vascular risk factors included cardiovascular disease (myocardial infarction, intermittent claudication, angina, heart failure, or other evidence of coronary disease), dyslipidemia (low levels of high-density lipoprotein cholesterol, high levels of low-density lipoprotein 
cholesterol, or hypertriglyceridemia), type 2 diabetes, atrial fibrillation, evidence of carotid artery disease, and transient ischemic attack (TIA) or minor stroke.

\section{Neuropsychological battery}

Cognitive measures included (1) WMS-R Logical Memory II subtest, immediate and delayed recall on Story A; (2) Rey Auditory Verbal Learning Test (RAVLT), total immediate recall score for trials 1-5, delayed recall score, and recognition score; (3) Wechsler Adult Intelligence ScaleRevised Digit Span forward and backward scores and spans; (4) Trail Making Tests A and B, times to completion; (5) Animal Fluency total score; (6) Vegetable Fluency total score; and (7) Boston Naming Test (BNT) total score (see Table 2).

\section{Brain volume estimation}

Participants had magnetic resonance imaging (MRI) at 1.5 T. The data were obtained following a standardized MRI protocol (http://adni.loni.usc.edu/about/centers-cores/ mri-core/) that was developed following an initiative to evaluate and compare three-dimensional T1-weighted sequences for morphometric analyses [36]. Each participant had two T1-weighted MRI scans collected using a sagittal volumetric magnetization prepared rapid gradient echo sequence with the following acquisition parameters: echo time of 4 milliseconds, repetition time of 9 milliseconds, flip angle of 8 degrees, acquisition matrix size of $256 \times 256 \times 166(x-, y$-, and $z$-dimensions $)$. The normal voxel size was $0.94 \times 0.94 \times 1.2 \mathrm{~mm}$.

Anatomical boundaries of the hippocampi and ventricles were traced using a semiautomated brain-mapping method developed on the basis of a high-dimensional fluid transformation algorithm [37]. White matter hyperintensities (WMH) were detected on coregistered T1-, T2-, and proton density-weighted images using automated methods described elsewhere [38, 39]. To correct for observed kurtosis in the distribution of the WMH volumes, we applied a log transformation prior to analyses.

\section{Statistical analyses \\ Cross-sectional analyses}

Data were screened for departures from normality using indices of skewness and kurtosis. Scores from the Trail Making Test A and B and the BNT exhibited significant skewness, which was corrected by log transformation. Groups were compared on clinical and demographic variables using chi-square tests for nominal variables and one-way analysis of variance (ANOVA) for continuous variables. Performance on neuropsychological measures, as well as measures of hippocampal, ventricular, and WMH volume were compared using one-way ANOVA and analysis of covariance, controlling for age, sex, education, APOE \&4 allele carrier status, and BMI. Both analyses produced the same pattern of results; hence, only corrected analyses are presented in Table 2. Group differences were examined using post hoc least significant difference tests and chi-square analyses. All analyses were two-tailed, with significance set at $p<0.05$.

\section{Longitudinal analyses}

To examine group differences and time $\times$ group interactions for cognitive performance and brain volume measures, multilevel model analyses were conducted with compound symmetric covariance structure and restricted maximum likelihood estimation. Time was entered as a random effect. Group, time $\times$ group, age, sex, education, APOE $\varepsilon 4$ carrier status, and BMI were entered as fixed factors. Participants were clustered by site to account for local prescribing practice. Analyses were also conducted with the inclusion of systolic blood pressure and the number of antihypertensive medications taken at baseline as additional covariates. The broad pattern of results remained unchanged; hence, results without these two covariates are presented. Given that this was an exploratory study, with no prior studies having investigated the use of ARBs in relation to comprehensive measures of neuropsychological function, we did not apply multiple comparison corrections. Analyses were two-tailed with $\alpha$ set at $p<0.05$. All analyses were performed with IBM SPSS for Mac OS X version 21.0 (IBM, Armonk, NY, USA) and SAS version 9.4 (SAS Institute Inc., Cary, NC, USA) software.

\section{Results}

Physiological, clinical, and genetic data

Three groups were compared: ARB users (HTN-ARBs), users of other antihypertensive drugs (HTN-Other), and a normotensive group that did not take any antihypertensive drugs (see "Blood pressure medication groups" section above and Additional files 1 and 2 for the specific medications used per group). As shown in Table 1, there were significant group differences in sex and diastolic blood pressure $(p<0.05)$, age, BMI, systolic blood pressure, pulse pressure (all $p<0.001)$, and education $(p=0.005)$. The normotensive group had significantly fewer males than the HTN-Other group $(p=0.004)$, was significantly younger $(p<0.05)$, and had a higher education level $(p<0.05)$. It also had better vascular health, as demonstrated by significantly lower diastolic blood pressure $(p<0.05)$, BMI, systolic blood pressure, and pulse pressure (all $p<0.001$ ), than both treated hypertensive groups. At the 3-year follow-up, normotensive individuals continued to have significantly lower systolic blood pressure than both hypertensive groups (HTN-ARBs $p=0.01$, HTN-Other $p<0.001)$ and lower pulse pressure than the HTNOther group $(p<0.001)$. 
Table 1 Clinical and demographic data

\begin{tabular}{|c|c|c|c|c|c|c|}
\hline Characteristics & $\begin{array}{l}\text { Total } \\
(n=1586)\end{array}$ & $\begin{array}{l}\text { Normotensive } \\
(n=782)\end{array}$ & $\begin{array}{l}\text { HTN-ARBs } \\
(n=183)\end{array}$ & $\begin{array}{l}\text { HTN-Other } \\
(n=621)\end{array}$ & F or $x^{2}$ value & $p$ Value \\
\hline \multicolumn{7}{|l|}{ Baseline clinical/demographic characteristics } \\
\hline Age, years & $73.1(7.2)$ & $71.9(7.3)$ & $73.3(6.8)$ & $74.6(6.8)$ & 25.737 & $<0.001$ \\
\hline Education, years & $16.1(2.8)$ & $16.3(2.7)$ & $15.9(3.2)$ & $15.9(2.8)$ & 5.282 & 0.005 \\
\hline Male sex, \% & $53.8 \%$ & $50.6 \%$ & $51.6 \%$ & $58.4 \%$ & 8.685 & 0.013 \\
\hline APOE genotype, $\varepsilon 4$ allele-positive, $\%$ & $42.3 \%$ & $43.4 \%$ & $36.8 \%$ & $42.5 \%$ & 2.365 & 0.306 \\
\hline $\mathrm{MCl}$ diagnosis, $\%$ & $60.3 \%$ & $59.8 \%$ & $54.4 \%$ & $62.7 \%$ & 4.216 & 0.121 \\
\hline $\mathrm{BMI}, \mathrm{kg} / \mathrm{m}^{2}$ & $27.1(4.8)$ & $26.4(4.6)$ & $28.0(4.8)$ & $27.6(4.8)$ & 14.844 & $<0.001$ \\
\hline Systolic BP, mmHg & $134.1(15.7)$ & $130.9(14.4)$ & $138.5(16.4)$ & $136.7(16.2)$ & 32.318 & $<0.001$ \\
\hline Diastolic BP, mmHg & $74.5(9.4)$ & $73.9(8.9)$ & $75.5(9.7)$ & $75.0(9.8)$ & 3.467 & 0.031 \\
\hline Pulse pressure, mmHg & $59.6(14.2)$ & $57.1(13.1)$ & $63.0(15.5)$ & $61.7(14.6)$ & 25.014 & $<0.001$ \\
\hline \multicolumn{7}{|l|}{ Baseline vascular risk factors } \\
\hline Cardiovascular disease & $11.2 \%$ & $3.7 \%$ & $17.6 \%$ & $18.8 \%$ & 87.645 & $<0.001$ \\
\hline Dyslipidemia & $46.2 \%$ & $37.6 \%$ & $59.3 \%$ & $53.2 \%$ & 48.249 & $<0.001$ \\
\hline Type 2 diabetes & $8.6 \%$ & $3.7 \%$ & $23.1 \%$ & $10.5 \%$ & 75.236 & $<0.001$ \\
\hline Atrial fibrillation & $3.3 \%$ & $1.8 \%$ & $3.3 \%$ & $5.1 \%$ & 12.293 & 0.002 \\
\hline Carotid artery disease & $0.6 \%$ & $0.4 \%$ & $0.5 \%$ & $1.0 \%$ & 1.888 & 0.389 \\
\hline $\mathrm{TIA} /$ minor stroke & $2.9 \%$ & $1.3 \%$ & $3.3 \%$ & $4.8 \%$ & 15.569 & $<0.001$ \\
\hline 3-Year follow-up blood pressure data & $\begin{array}{l}\text { Total } \\
(n=779)\end{array}$ & $\begin{array}{l}\text { Normotensive } \\
(n=380)\end{array}$ & $\begin{array}{l}\text { HTN-ARBs } \\
(n=89)\end{array}$ & $\begin{array}{l}\text { HTN-Other } \\
(n=310)\end{array}$ & & \\
\hline Systolic BP, mmHg & $131.9(16.9)$ & $129.0(15.8)$ & $133.9(16.2)$ & $134.9(17.7)$ & 11.602 & $<0.001$ \\
\hline Diastolic BP, mmHg & $72.6(9.6)$ & $72.0(9.3)$ & $74.0(10.2)$ & $73.0(9.8)$ & 1.843 & 0.159 \\
\hline Pulse pressure, $\mathrm{mmHg}$ & $59.3(14.7)$ & $56.9(14.0)$ & $59.9(13.2)$ & $61.9(15.5)$ & 10.203 & $<0.001$ \\
\hline
\end{tabular}

Abbreviations: APOE Apolipoprotein E, BMI Body mass index, BP Blood pressure, HTN-ARBs Participants who took angiotensin II receptor blockers, HTN-Other Participants who took other antihypertensive drugs that were not angiotensin II receptor blockers, MCI Mild cognitive impairment, TIA Transient ischemic attack Data are summarized as mean (SD), unless otherwise indicated

Significant differences $(p<0.05)$ among medication groups are indicated by boldface type

\section{Vascular risk factors}

There were significant group differences in history of cardiovascular disease, dyslipidemia, type 2 diabetes, TIA/minor stroke (all $p<0.001$ ), and atrial fibrillation $(p=0.002)$. Both hypertensive groups had significantly more participants with prior cardiovascular disease $(p<$ $0.001)$, dyslipidemia $(p<0.001)$, and type 2 diabetes $(p<0.001)$ than the normotensive group. The HTNOther group also had significantly more participants with a history of atrial fibrillation $(p<0.001)$ and TIA/stroke $(p<0.001)$ than the normotensive group. The ARB group had significantly more participants with type 2 diabetes than the HTN-Other group $(p<0.001)$.

\section{Cross-sectional analyses}

\section{Neuropsychological function}

As shown in Table 2, there were significant group differences on measures of memory, attention, and executive function after correcting for covariates. The HTN-Other group performed worse than the normotensive group on all of these measures (see Fig. 1): RAVLT Immediate Recall $(p=0.002)$, RAVLT Delayed Recall $(p<0.001)$, RAVLT Recognition $(p=0.001)$, Trail Making Test A $(p<$ $0.001)$, and Trail Making Test B $(p=0.01)$. The HTNARBs group performed worse than the normotensive group only on Trail Making Test A $(p=0.04)$. The HTN-ARBs group performed significantly better than the HTN-Other group on a measure of memory (RAVLT Recognition $p=0.04$ ) and displayed a nonsignificant trend toward outperforming the HTN-Other group on RAVLT Delayed Recall $(p=0.058)$.

\section{Brain MRI measures}

There were significant group differences in WMH volume $\left[F(2,1252)=4.41, p=0.01, \eta_{\mathrm{p}}^{2}=0.01\right]$ from the HTN-Other group exhibiting significantly greater WMH volume than the normotensive group ( $p=$ $0.004)$. There were no differences in ventricular volume $[F(2,552)=0.22, p=0.81]$, left hippocampal volume 
Table 2 Baseline neuropsychological data

\begin{tabular}{|c|c|c|c|c|c|c|}
\hline Neuropsychological test & Total $(n=1586)$ & Normotensive $(n=782)$ & HTN-ARBs $(n=183)$ & HTN-Other $(n=621)$ & F-Value & $p$ Value \\
\hline \multicolumn{7}{|l|}{ Memory } \\
\hline LM Immediate Recall & $10.6(4.3)$ & $10.7(4.4)$ & $10.9(4.3)$ & $10.4(4.2)$ & 0.269 & 0.764 \\
\hline LM Delayed Recall & $8.5(5.0)$ & $8.6(5.1)$ & $9.2(5.0)$ & $8.3(4.8)$ & 1.987 & 0.137 \\
\hline RAVLT Immediate Recall & $38.2(11.6)$ & $39.6(11.8)$ & $38.1(11.8)$ & $36.5(11.1)$ & 4.986 & 0.007 \\
\hline RAVLT Delayed Recall & $5.3(4.3)$ & $5.8(4.4)$ & $5.5(4.4)$ & $4.5(3.9)$ & 9.642 & $<0.001$ \\
\hline RAVLT Recognition & $11.4(3.3)$ & $11.7(3.2)$ & $11.7(3.1)$ & $11.0(3.5)$ & 5.692 & 0.003 \\
\hline \multicolumn{7}{|l|}{ Attention/executive function } \\
\hline Digit Span forward score & $8.4(2.0)$ & $8.5(1.9)$ & $8.1(2.2)$ & $8.4(2.1)$ & 1.100 & 0.402 \\
\hline Digit Span forward span & $6.6(1.1)$ & $6.7(1.0)$ & $6.5(1.1)$ & $6.6(1.1)$ & 0.953 & 0.386 \\
\hline Digit Span backward score & $6.5(2.1)$ & $6.6(2.2)$ & $6.5(2.1)$ & $6.4(2.1)$ & 0.121 & 0.886 \\
\hline Digit Span backward span & $4.8(1.2)$ & $4.8(1.2)$ & $4.7(1.1)$ & $4.7(1.2)$ & 0.021 & 0.979 \\
\hline Trail Making Test A score ${ }^{a}$ & $1.56(0.16)$ & $1.53(0.15)$ & $1.58(0.16)$ & $1.58(0.16)$ & 8.363 & $<0.001$ \\
\hline Trail Making Test B score ${ }^{a}$ & $1.97(0.21)$ & $1.94(0.21)$ & $1.98(0.20)$ & $2.00(0.21)$ & 3.900 & 0.020 \\
\hline \multicolumn{7}{|l|}{ Language } \\
\hline Animal Fluency & $18.4(5.5)$ & $18.7(5.4)$ & $18.4(5.3)$ & $18.0(5.7)$ & 0.173 & 0.841 \\
\hline Vegetable Fluency & $12.2(4.1)$ & $12.3(3.9)$ & $12.2(4.2)$ & $12.1(4.3)$ & 0.206 & 0.814 \\
\hline$B N T^{a}$ & $0.48(0.33)$ & $0.47(0.33)$ & $0.47(0.33)$ & $0.51(0.33)$ & 1.385 & 0.251 \\
\hline
\end{tabular}

Abbreviations: BNT Boston Naming Test, HTN-ARBs Participants who took angiotensin II receptor blockers, HTN-Other Participants who took other antihypertensive drugs that were not angiotensin II receptor blockers, LM Logical Memory, RAVLT Rey Auditory Verbal Learning Test

Data are summarized as mean (SD), unless otherwise indicated. All scores were corrected for age, sex, education level, BMI, and apolipoprotein $\varepsilon 4$ allele carrier status. Significant differences $(p<0.05)$ among medication groups are indicated by boldface type

${ }^{a}$ Scores were log-transformed and are presented to two decimal places

$[F(2,552)=0.48, p=0.62]$, or right hippocampal volume $[F(2,552)=0.93, p=0.40]$.

\section{Longitudinal analyses}

\section{Neuropsychological function}

There were significant time $\times$ group interactions for both measures of Logical Memory, Immediate Recall $[F(2,1772)=3.63, p=0.03]$, and Delayed Recall $[F(2$, $1767)=3.72, p=0.02$ ]. As shown in Fig. 2, the HTNOther group showed significantly worse performance on Immediate Recall over the 3-year follow-up than normotensive subjects $[\beta=-0.22, t(1772)=-2.39, p=$ 0.02 ], as well as compared with the HTN-ARBs group $[\beta=-0.29, t(1772)=-2.02, p=0.04]$. The HTN-ARBs group was no different from the normotensive group $[\beta=0.07, t(1772)=0.48, p=0.63]$.

As shown on Fig. 3, for Logical Memory Delayed Recall, the HTN-Other group performed worse than normotensive subjects over the follow-up period $[\beta=-0.27$, $t(1767)=-2.72, p=0.007]$. The HTN-ARBs group was no different from normotensive subjects $[\beta=-0.08$, $t(1767)=-0.57, p=0.57]$.

There were significant effects of group for RAVLT Immediate Recall $[F(2,3006)=3.61, p=0.03]$, RAVLT Delayed Recall $[F(2,2995)=5.94, p=0.003]$, RAVLT Recognition $[F(2,2991)=3.23, p=0.04]$, Trail Making Test A $[F(2,2998)=7.63, p=0.0005]$, and Trail Making
Test B performance $[F(2,2932)=4.51, p=0.01]$. The HTN-Other group performed significantly worse than the normotensive subjects on all these measures (RAVLT Immediate Recall $p=0.008$, RAVLT Delayed Recall $p=$ 0.0006, RAVLT Recognition $p=0.03$, Trail Making Test A $p=0.0007$, Trail Making Test B $p=0.006)$. The HTNARBs group performed worse than normotensive subjects on Trail Making Test A $(p=0.004)$ and Trail Making Test $\mathrm{B}(p=0.04)$ only. The results were no different from those of normotensive subjects on all RAVLT measures.

\section{Brain MRI measures}

There were significant main effects of time for WMH volume $[F(1,2277)=32.11, p<0.0001]$, left hippocampal volume $[F(1,1059)=1245.07, p<0.0001]$, right hippocampal volume $[F(1,1059)=1664.23, p<0.0001]$, and ventricular volume $[F(1,1059)=1045.76, p<0.0001]$. Ventricular volume and WMH volume significantly increased over time, whereas both left and right hippocampal volume significantly decreased over time (all $p<$ 0.0001). There were no significant interactions between time and group and no significant main effects of group. However, there was a nonsignificant group trend for WMH volume $[F(2,2279)=2.61, p=0.07]$. The HTNOther group showed a nonsignificant trend toward having greater WMH volume than the HTN-ARBs group $(p=0.08)$ and normotensive subjects $(p=0.05)$. 


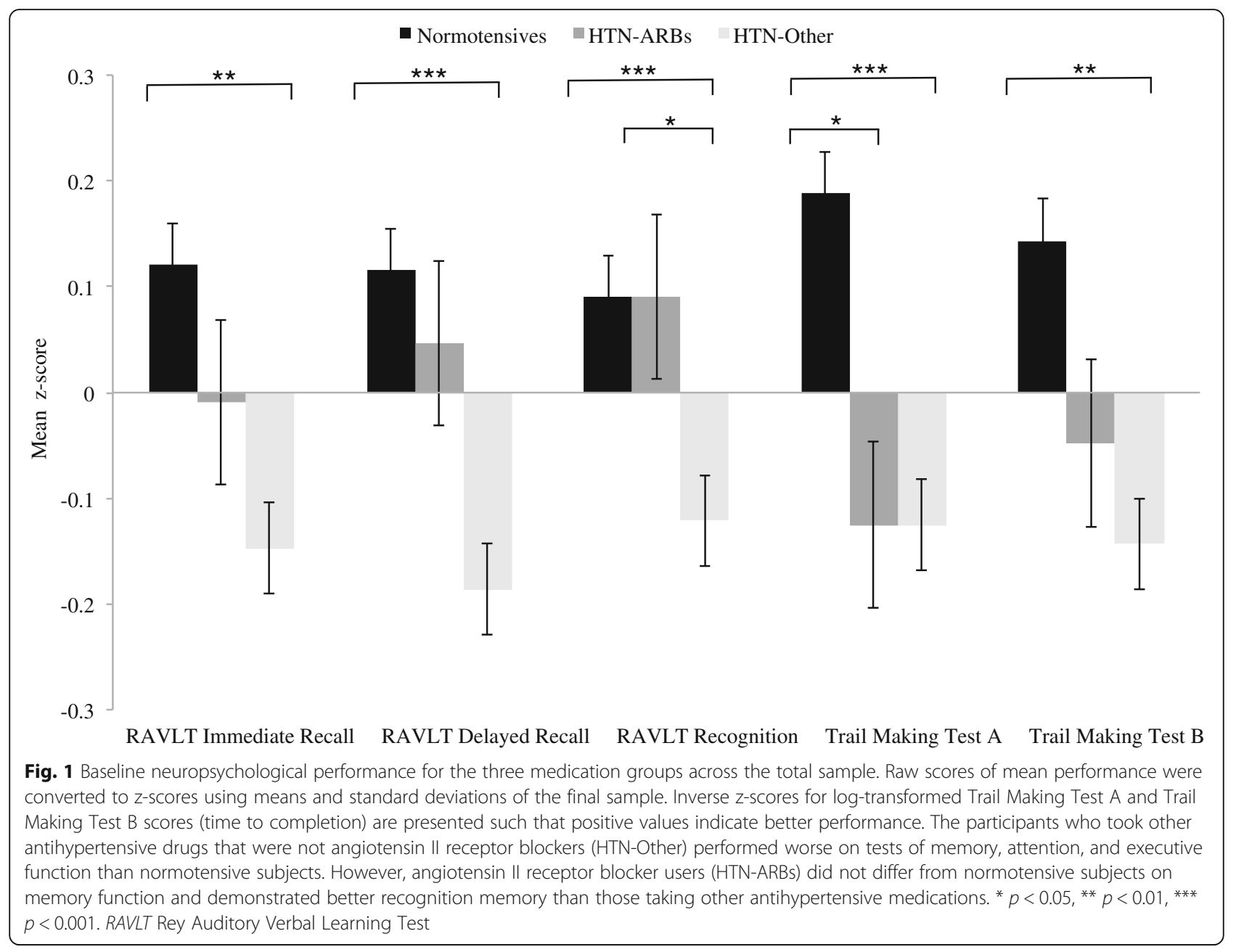

\section{Post hoc exploratory analyses}

\section{Drugs affecting the renin-angiotensin-aldosterone-system}

In post hoc multilevel model analyses, we examined whether users of RAAS drugs (ACEIs and ARBs) performed as well as the HTN-ARBs group. When this group (the ARB-ACEI group) was compared with users of other antihypertensive drugs (HTN-Other) and normotensive subjects, a significant time $\times$ group interaction was found for Logical Memory Delayed Recall $[F(2,1767)=3.00$, $p=0.05]$. The ARB-ACEI group performed worse than normotensive subjects on this measure over time $[\beta=-0.23$, $t(1767)=-2.17, p=0.03]$. There was a trending effect for the HTN-Other group performing worse than normotensive subjects $[\beta=-0.22, t(1767)=-1.81, p=0.07]$.

There were significant effects of group for RAVLT Immediate Recall $[F(2,3006)=3.46, p=0.03]$, RAVLT Delayed Recall $[F(2,2995)=5.07, p=0.006]$, Digit Span Forward Score $[F(2,1471)=3.32, p=0.04]$, Digit Span Forward Span $[F(2,1466)=2.91, p<0.05]$, and $\mathrm{WMH}$ volume $[F(2,2277)=4.52, p=0.01]$. Both the ARB-ACEI group and the HTN-Other group performed worse than normotensive subjects on the RAVLT measures (Immediate
Recall both $p=0.03$, Delayed Recall both $p<0.01)$. The ARB-ACEI group also performed worse than normotensive subjects on Digit Span Forward Score and Digit Span Forward Span (both $p=0.02$ ). They performed worse than the HTN-Other group on Digit Span Forward Score $(p=0.03)$. Both the ARB-ACEI and the HTN-Other groups demonstrated greater WMH volume than normotensive subjects (ARB-ACEI $p=0.005$, HTN-Other $p=0.04$ ).

\section{BBB-crossing capability}

Given that the capability of medications to cross the $\mathrm{BBB}$ is related to drug efficacy, a group using BBBcrossing ARBs (ARB crossers) was compared with a group using non-BBB-crossing (ARB noncrossers), a group using all other antihypertensive medications (HTNOther), and normotensive subjects. Significant time $\times$ group interactions were found for three measures of memory: RAVLT Delayed Recall $[F(3,1759)=5.07, p=0.002]$, Logical Memory Immediate Recall $[F(3,1772)=3.05$, $p=0.03]$, and Logical Memory Delayed Recall [ $F(3$, $1766)=3.39, p=0.02$ ] The HTN-Other group performed significantly worse over time than both the 


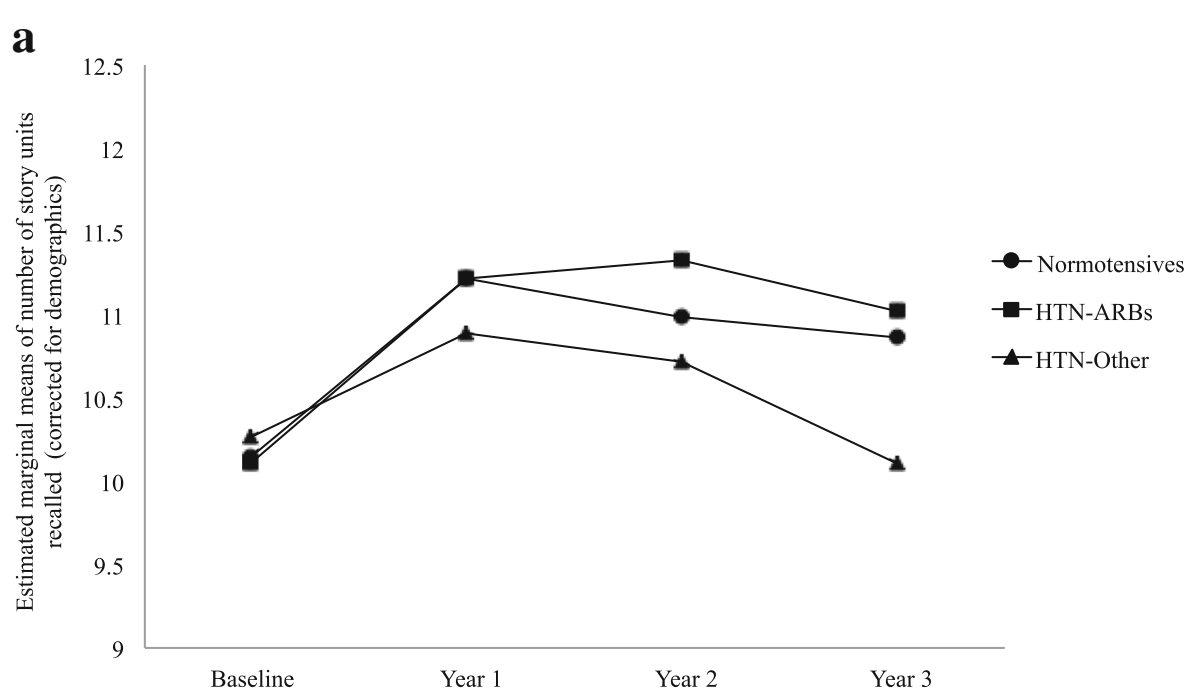

b

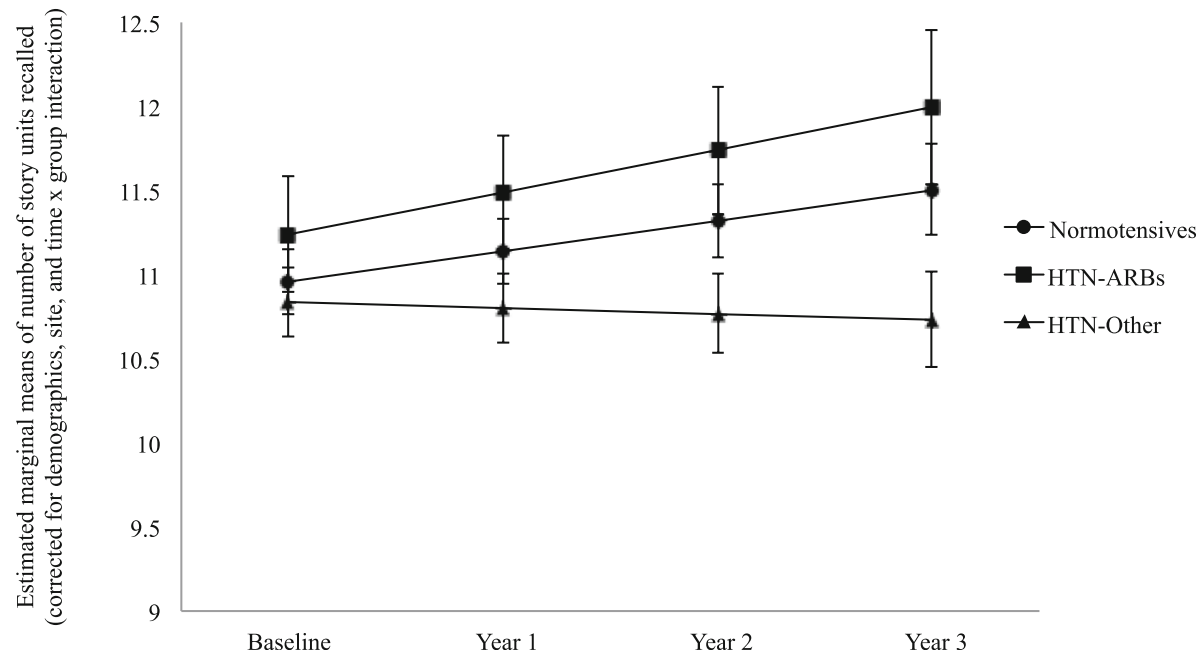

Fig. 2 Logical Memory Immediate Recall performance over the 3-year follow-up period. a Estimated marginal means after correction for demographics only. b Means adjusted for demographics, Alzheimer's Disease Neuroimaging Initiative site, and time $\times$ group interaction. After all adjustments, the participants who took other antihypertensive drugs that were not angiotensin II receptor blockers (HTN-Other) showed declining performance over time that was significantly worse than that of normotensive subjects and the participants who took angiotensin II receptor blockers (HTN-ARBs), with both of the latter groups showing improvement. The HTN-ARBs group was no different from normotensive subjects

ARB crossers group and normotensive subjects on all three measures (all $p<0.05$ ). On RAVLT Delayed Recall, the ARB noncrossers group performed significantly worse than the HTN-Other group ( $p=0.005)$, the ARB crossers group ( $p=0.0003)$, and normotensive subjects $(p=0.03)$.

There were significant effects of group for RAVLT Recognition $[F(3,2988)=2.80, p=0.04]$ and $\mathrm{WMH}$ volume $[F(3,2275)=5.28, p=0.001]$. The HTN-Other group performed worse than the ARB noncrossers group $(p=0.02)$ and normotensive subjects $(p=0.03)$ on RAVLT Recognition. As shown on Fig. 4, the HTN-Other group also had significantly greater WMH volume than the ARB crossers group ( $p=0.008)$ and normotensive subjects $(p=0.0006)$. Within the ARB user group, the ARB crossers group had less WMH volume than the ARB-noncrossers group $(p=0.05)$.

\section{BBB-crossing RAAS medications}

A group using BBB-crossing RAAS medication users (BBB crossers) was compared with a group using nonBBB-crossing RAAS medications (BBB noncrossers), a group of users of all other antihypertensive medications (HTN-Other), and normotensive subjects. Significant time $\times$ group interactions were found for two measures 

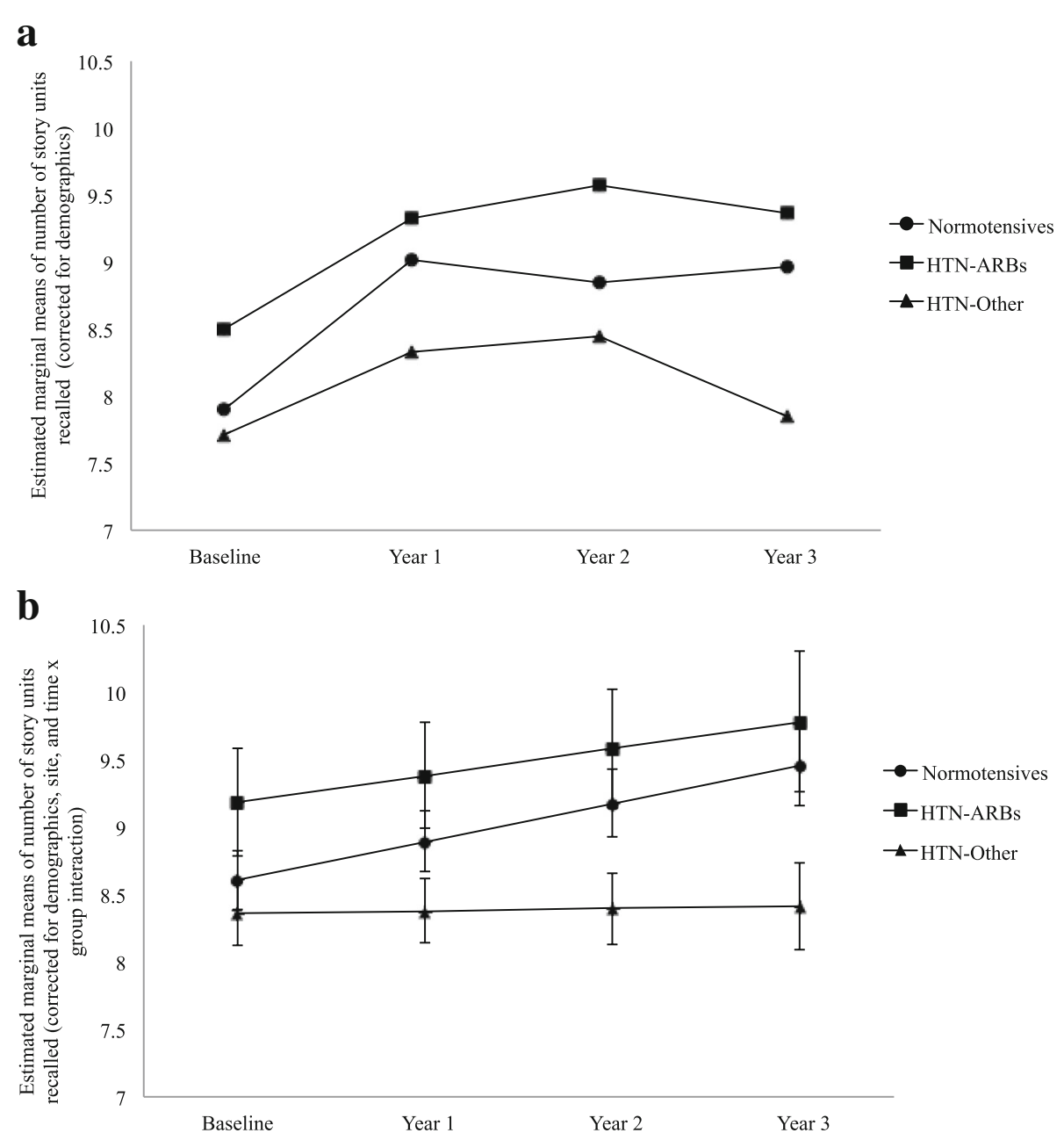

Fig. 3 Logical Memory Delayed Recall performance over the 3-year follow-up period. a Estimated marginal means after correction for demographics only. b Means adjusted for demographics, Alzheimer's Disease Neuroimaging Initiative site, and time $\times$ group interaction. After all adjustments, the participants who took other antihypertensive drugs that were not angiotensin II receptor blockers (HTN-Other) showed stable performance over time that was significantly worse than the performance of normotensive subjects, who showed improvement over time. The participants who took angiotensin II receptor blockers (HTN-ARBs) also improved over time and were no different from normotensive subjects

of memory-RAVLT Delayed Recall $[F(3,1752)=4.58$, $p=0.003]$ and Logical Memory Delayed Recall [F(3, $1759)=2.66, p=0.05]$ - as well as for WMH volume $[F(3,1135)=2.60, p=0.05]$. Users of BBB-crossing RAAS medications performed better than all other groups, including normotensive subjects, on RAVLT Delayed Recall over time (BBB noncrossers and normotensive subjects $p=$ 0.001 , HTN-Other $p=0.01)$. Additionally, they were no different from normotensive subjects on Logical Memory Delayed Recall over time $(p=0.14)$, unlike the BBB noncrossers group, which performed significantly worse than normotensive subjects $(p=0.02)$. Users of other antihypertensive medications showed a trend toward performing worse than normotensives on this measure $(p=0.07)$. The BBB crossers group also had more WMH volume over time than normotensive subjects $(p=0.01)$ but less WMH volume than the BBB-noncrossers group $(p=0.03)$.

\section{Discussion}

Findings of the present study provide support for our hypothesis that ARBs may confer protective effects on cognition greater than those of other antihypertensive drugs. At baseline, participants taking other antihypertensive medications exhibited worse memory and executive function than normotensive subjects, but ARB users did not differ from normotensive subjects on any measure of memory function and demonstrated better recognition memory than those taking other antihypertensive medications. Over a 3-year follow-up period, those taking other antihypertensive drugs performed significantly worse than normotensive individuals on immediate and delayed recall of a short story, but ARB users were no different from normotensive individuals on memory testing. These cognitive differences are underscored by the fact that both hypertensive groups exhibited elevated vascular risk factor 

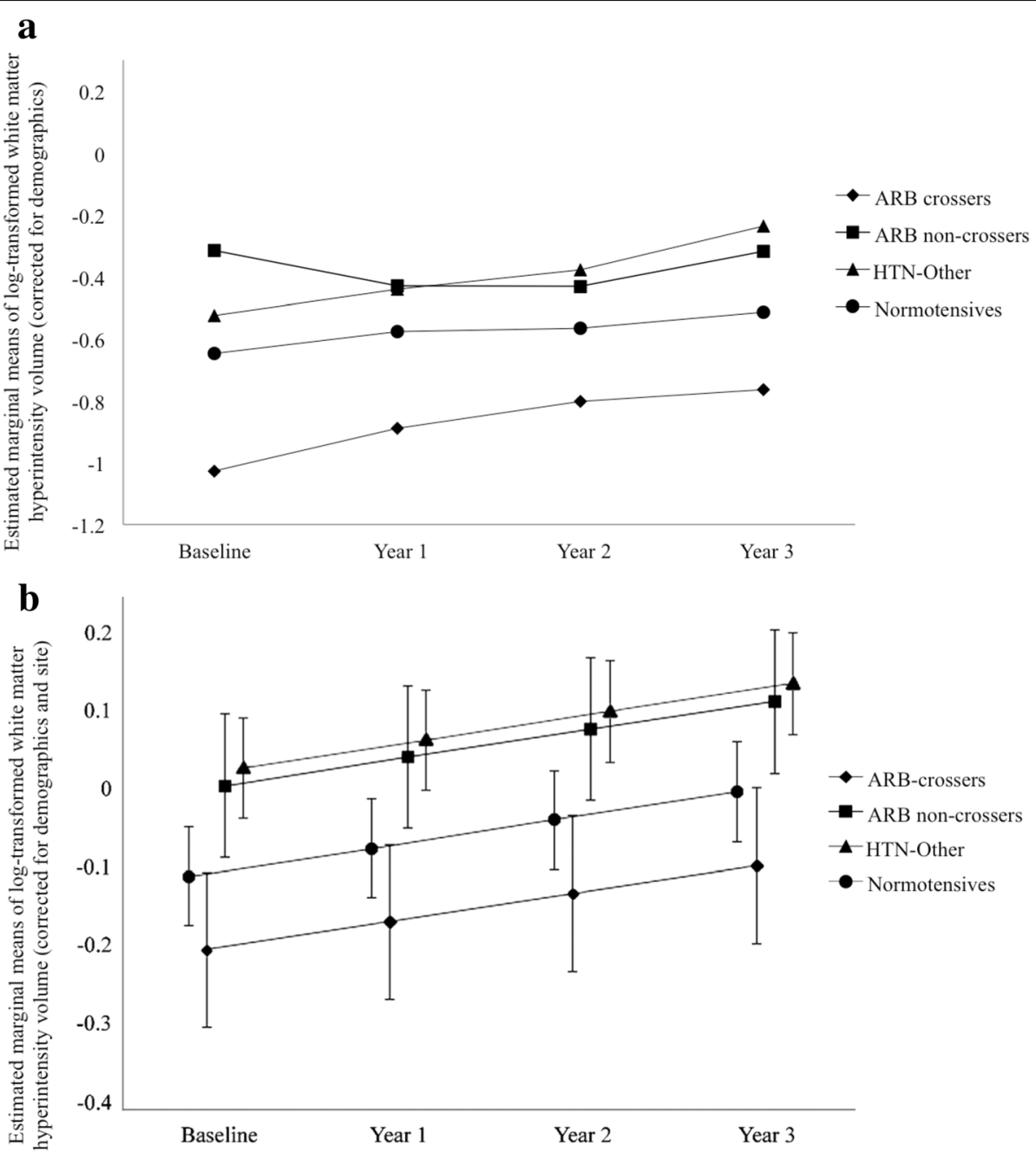

Fig. 4 White matter hyperintensity (WMH) volume over the 3-year follow-up period. a estimated marginal means after correction for demographics only, $\mathbf{b}$ means adjusted for demographics and Alzheimer's Disease Neuroimaging Initiative site. After all corrections, the participants who took other antihypertensive drugs that were not angiotensin II receptor blockers (HTN-Other) showed significantly greater WMH volume over time compared with users of blood-brain barrier (BBB)-crossing angiotensin II receptor blocker users (ARBs; BBB crossers) and normotensive subjects. Within the ARB users, the BBB crossers group had less WMH volume than users of non-BBB-crossing ARBs

burden relative to the normotensive group, as evidenced in higher systolic and diastolic blood pressure, pulse pressure, and BMI scores, as well as prior diagnoses of cardiovascular disease, dyslipidemia, and type 2 diabetes. Therefore, the hypertensive groups as a whole were potentially more vulnerable to cognitive impairment, but only the subset taking ARBs demonstrated expected practice effects on memory testing. The cognitive differences between the medicated hypertensive groups are especially notable, given that the ARB group had significantly more participants diagnosed with type 2 diabetes, which has been associated with a 1.5- to 2.5 -fold greater risk of dementia [40]. Even with this added risk factor, the ARB users demonstrated preserved memory function over 3 years of follow-up.
Consistent with prior work suggesting that ACEIs may be less beneficial than ARBs for cognition, in the present study we found that when ARB users were combined with users of ACEIs, beneficial effects on memory were no longer observed. Li and colleagues [41], who examined 819,491 predominantly male participants over the age of 65 years with cardiovascular disease in a large epidemiological study, reported that ARB use was associated with a significant reduction in the incidence and progression of dementia and Alzheimer's disease in comparison to ACEIs and other cardiovascular drugs. Additionally, researchers in a nested case-control study of adults aged 60 years or older, who had either Alzheimer's disease, vascular dementia, or unspecified/other dementia, reported that patients taking ARBs and ACEIs had 53\% and 24\% lower 
risks of Alzheimer's disease, respectively, than those taking other antihypertensive medications [42]. On one hand, whereas ACEIs reduce the amount of free angiotensin II and decrease damaging angiotensin II receptor type 1 (AT1) receptor activity, they also reduce beneficial angiotensin II receptor type 2 (AT2) receptor activity. On the other hand, given that ARBs block AT1 receptors and not the production of angiotensin II, they promote AT2 receptor activity and are "angiotensin-converting enzyme (ACE)-sparing," in theory allowing ACE to continue its suggested $A \beta$-degrading function, unlike ACEIs [43].

Nevertheless, it must be noted that investigators in large clinical intervention trials such as ONTARGET and TRANSCEND have not found significant differences in the incidence of dementia between groups taking ARBs, ACEIs, or placebo [44]. Reasons for the discrepancies in these results and the results of the present study may include differences in sample characteristics. In both ONTARGET and TRANSCEND, researchers examined patients with high vascular disease burden, evidenced by established atherosclerotic cardiovascular disease or diabetes with end-organ damage. Such patients are likely to develop dementia with vascular etiology. In contrast, participants in the ADNI sample were excluded on the basis of criteria that limit cerebrovascular disease. Hence, the ratio of vascular to Alzheimer's disease pathology was likely greater in the samples in ONTARGET and TRANSCEND. The lack of distinction between dementia subtypes in these studies may have obscured any beneficial effects that hypertensive treatment may have had on specific types of dementia, which is important, given the differential effects of ARBs and ACEIs on A $\beta$ and Alzheimer's disease pathology. Additionally, the mean ages of participants in ONTARGET and TRANSCEND were 66.4 and 66.9 years, respectively, which are 7-8 years younger than the mean age of the patients with hypertension in our sample and approximately a decade younger than the age at which rates of dementia increase [44]. Because Alzheimer's disease risk increases with age, our sample likely had greater Alzheimer's disease pathology, which may have contributed to our results of group differences in memory testing. The intervention trials also relied heavily on achieved scores and changed scores on a single measure (MMSE) to diagnose cognitive impairment and cognitive decline. Our study benefits from assessment of cognition through a comprehensive neuropsychological battery that may have been better suited to discerning changes across a range of domains.

Our study also found associations between the use of BBB-crossing ARBs and better performance across the 3-year follow-up on three measures of memory (RAVLT Delayed Recall, Logical Memory Immediate Recall, and Logical Memory Delayed Recall) in comparison to the HTN-Other group, which in turn performed worse than normotensive subjects. Interestingly, on the first measure, users of BBB-crossing ARBs performed better than users of non-BBB-crossing ARBs. Group differences were also found in WMH volume, with users of BBB-crossing ARBs showing significantly less WMH volume than all other treated subjects with hypertension. A combined group of users of BBB-crossing ARBs and BBB-crossing ACEIs demonstrated better performance on delayed list-learning recall over a 3-year follow-up period than users of non-BBB-crossing ARBs and ACEIs and had less WMH volume than this group. Therefore, among the various antihypertensive medications affecting the RAAS, those with the capacity to cross the BBB were associated with better memory over time and less WMH volume than their noncentrally acting counterparts. However, our findings were somewhat mixed with regard to memory because users of non-BBB-crossing ARBs showed better memory on some test scores, suggesting that among ARBs, both the BBBcrossing and non-BBB-crossing varieties may be of benefit.

Prior work has similarly examined differences in cognition among users of BBB-crossing and non-BBB-crossing ACEIs. Ohrui and colleagues [45], in examining differences among hypertensive patients taking BBB-crossing ACEIs (captopril or perindopril) and patients taking non-BBBcrossing ACEIs (imidapril or enalapril), calcium channel blockers, $\beta$-blockers, and diuretics, reported significantly lower risk of Alzheimer's disease in the group taking $\mathrm{BBB}$-crossing ACEIs than in those taking the non-BBBcrossing variety (odds ratio $0.25,95 \%$ confidence interval $0.08-0.75, p=0.014)$. Other studies have also found associations between BBB-crossing ACEIs and less severe cognitive decline in MMSE and modified MMSE scores when compared with non-BBB-crossing ACEI use $[45,46]$. Nevertheless, these results need confirmation in an RCT of BBB-crossing hypertensive drugs in dementia prevention or reduction.

The protective mechanism responsible for preserved memory among ARB users remains uncertain and is likely to involve a complex interaction of multiple pathways. In the present study, only BBB-crossing ARB users showed significantly lower WMH volume relative to other antihypertensive medication users, who exhibited greater white matter lesion burden than normotensive subjects. Notably, when ARB users were combined with users of ACEIs, beneficial effects on memory and WMH volume disappeared: The combined ARB-ACEI group, as well as users of all other antihypertensive drugs, performed worse on measures of immediate and delayed recall and had greater WMH volume than normotensive subjects. The ARB-ACEI group also performed worse than normotensive subjects on measures of attention. Therefore, compared with other drugs impacting the RAAS, ARBs appear to be associated with maintenance of memory and attention, as well as amount of white matter lesions, which can disrupt 
frontal-subcortical connections critical to memory retrieval functions [47].

ARBs may also exert their effects through improving cerebral blood flow and reducing ischemia through their influence on the RAAS. The RAAS regulates blood pressure through its effects on fluid homeostasis and vascular tone. Although the RAAS is more commonly associated with endocrine and vascular-renal functions, research has also established the presence of a paracrine RAAS within the central nervous system that acts largely independently of peripheral function [48]. The RAAS in the brain is believed to be involved in processes beyond mere blood pressure control, including processes of learning and memory [49]. ARBs target the locally acting brain RAAS and block AT1 receptors, allowing greater AT2 receptor binding [32], thus exerting their effects by both interrupting AT1 receptor activity and promoting AT2 receptor activity. AT1 receptor activity includes the generation of free radicals and the activation of multiple inflammatory pathways, all of which lead to tissue damage [50]. Greater AT2 receptor activity decreases vasoconstriction, thus increasing cerebral blood flow, which is protective against inflammation and ischemia [51].

In a recent longitudinal analysis of cerebrospinal fluid biomarker data from ADNI, we reported an attenuation of cerebral amyloid retention and progression to dementia among older adults taking ARBs [52]; however, there were no cross-sectional differences in amyloid retention. The present study extends these findings by demonstrating maintenance of memory function and amount of WMH among ARBs users at baseline and 3-year follow-up. Together, these results present a complex picture in which ARBs may be protective of memory function by possibly stymieing effects of cerebral small vessel disease and/or Alzheimer's disease pathology or otherwise improving neuronal function through direct actions on cerebral blood flow or neuronal RAAS receptors. The strength of these benefits may vary, depending on the use of other drugs impacting RAAS, whether the ARBs cross the $\mathrm{BBB}$, and other individual differences such as age and vascular risk factor burden.

To our knowledge, this is the first study to examine performance on a comprehensive neuropsychological battery among users of various antihypertensive drugs, as well as the first study to investigate differences in cognitive trajectories among users of BBB-crossing and non-BBB-crossing ARBs. Our study benefits from the examination of performance on multiple tests measuring multiple cognitive domains. Other strengths of the present study are its longitudinal design, large sample size, and inclusion of neuroimaging markers of brain atrophy and WMH volume.

Despite these strengths, the present study is not without limitations. We did not account for multiple drug combinations, owing to sample size limits. We focused on the use of ARBs and were unable to fully disentangle the potential effects of ACEIs, independent of ARBs, owing to sample size limitations. Future studies are needed to determine the independent effects of ACEIs. Given the exploratory nature of this study, we did not account for multiple comparisons. Comparison of the baseline characteristics of participants who presented vs. those who did not present for the 3-year follow-up revealed that those who presented had significantly more MCI diagnoses, higher systolic and diastolic blood pressure, and higher rates of carotid artery disease (see Additional file 4). Therefore, participants who presented at follow-up may have had more concerns regarding their cognitive functioning and vascular health and may have biased results against a null effect. Owing to inconsistent recording of medication and medical history following baseline visits, it was impossible to determine whether participants switched between groups over time. Additionally, the ADNI sample is comprised of participants from over 50 sites in the United States and Canada with varied sampling biases. Thus, we accounted for local prescribing practice by clustering participants by site in our analyses. Participants were excluded on the basis of criteria that restricted cerebrovascular disease, limiting generalizability. Effects of white matter disease may be greater in the broader population. Further, we cannot rule out possible confounding by indication, because ARB use in itself may be a risk indicator for the severity of hypertension. ARBs are prescribed as supplementary drugs in older patients with hypertension that remains uncontrolled after use of other drugs [53]. However, if there had been confounding by indication, we would predict that the ARB users would demonstrate worse cognitive performance than the group taking other antihypertensive drugs, given that the ARB users had potentially more severe hypertension as well as a higher proportion of participants with type 2 diabetes. In light of this, the superior cognitive performance of the ARB group in comparison to users of other antihypertensive drugs, including those also impacting the RAAS, is therefore notable.

\section{Conclusions}

Hypertensive participants demonstrated worse baseline memory and executive function and greater memory decline over 3 years follow-up than normotensive subjects, unless they were ARB users, who showed preserved memory compared with those taking other antihypertensive drugs. Users of BBB-crossing ARBs showed superior memory performance over time compared with other antihypertensive drug users and had less WMH volume. Users of BBB-crossing medications (ARBs or ACEIs) showed better list-learning memory performance over time than all other groups, including normotensive subjects, and had less 
WMH volume over time than users of non-BBB-crossing medications. These findings demonstrate that ARBs, especially those of the BBB-crossing variety, are associated with greater memory preservation and less WMH volume than other antihypertensive medications.

\section{Additional files}

Additional file 1: Baseline medications. (DOC $29 \mathrm{~kb}$ )
Additional file 2: Baseline number of hypertensive medications. (DOC $29 \mathrm{~kb}$ )
Additional file 3: Flowchart of inclusion/exclusion of participants.
(PNG $272 \mathrm{~kb}$ )
Additional file 4: Baseline characteristics of participants with missing vs.
nonmissing data at 3-year follow-up visit. (DOC $92 \mathrm{~kb}$ )

\section{Abbreviations}

ACE: Angiotensin-converting enzyme; ACEl: Angiotensin-converting enzyme inhibitors; ADNI: Alzheimer's Disease Neuroimaging Initiative; ANOVA: Analysis of variance; APOE: Apolipoprotein E; ARB: Angiotensin II receptor blocker; AT1: Angiotensin II type 1; AT2: Angiotensin II type 2; Aß: Amyloid- $\beta$; BBB: Bloodbrain barrier; BMI: Body mass index; BNT: Boston Naming Test; BP: Blood pressure; HTN-ARB: Participants who took angiotensin II receptor blockers; HTNOther: Participants who took other antihypertensive drugs that were not angiotensin II receptor blockers; HYVT-COG: Hypertension in the Very Elderly Trial assessing cognitive decline and dementia incidence; LM: Logical Memory; MCl: Mild cognitive impairment; MMSE: Mini Mental State Examination; MRCElderly: Medical Research Council Elderly study; MRI: Magnetic resonance imaging; ONTARGET: Ongoing Telmisartan Alone and in Combination with Ramipril Global Endpoint Trial; PROGRESS: Perindopril Protection against Recurrent Stroke Study; RAAS: Renin-angiotensin-aldosterone system; RAVLT: Rey Auditory Verbal Learning Test; RCT: Randomized controlled trial; SHEP: Systolic Hypertension in the Elderly Program; SYST-EUR: Systolic Hypertension in Europe; TIA: Transient ischemic attack; TRANSCEND: Telmisartan Randomised AssessmeNt Study in ACE iNtolerant subjects with cardiovascular Disease; WAIS-R: Wechsler Adult Intelligence Scale-Revised; WMH: White matter hyperintensities; WMS-R: Wechsler Memory Scale-Revised

\section{Acknowledgements}

The authors are grateful to Geoffrey Corner for statistical consultation on this manuscript, to Alberto Jarrin-Lopez, John Madrigal, and Sophia Moritz for their assistance with coding, as well as to Natasha Williams for assistance with graphics. Data used in the preparation of this article were obtained from the Alzheimer's Disease Neuroimaging Initiative (ADNI) database (http://adni.loni.usc.edu). As such, the investigators within the ADNI contributed to the design and implementation of the ADNI and/or provided data but did not participate in the analysis or in the writing of this article. A complete listing of ADNI investigators can be found at http://adni.loni.usc.edu/wp-content/uploads/how_to_apply/ ADNI_Acknowledgement_List.pdf.

\section{Funding}

The study data analysis was supported by National Institutes of Health (NIH) grants (P50 AG005142 and P01 AG052350). Data collection and sharing for this project were funded by the Alzheimer's Disease Neuroimaging Initiative (ADNI) (NIH grant U01 AG024904) and by U.S. Department of Defense (DOD) ADNI award number W81XWH-12-2-0012. ADNI is funded by the National Institute on Aging, the National Institute of Biomedical Imaging and Bioengineering, and generous contributions from the following: AbbVie Alzheimer's Association; Alzheimer's Drug Discovery Foundation; Araclon Biotech; BioClinica, Inc.; Biogen; Bristol-Myers Squibb Company; CereSpir, Inc.; Eisai Inc.; Elan Pharmaceuticals, Inc.; Eli Lilly and Company; EUROIMMUN; F. Hoffmann-La Roche Ltd. and its affiliated company Genentech, Inc.; Fujirebio; GE Healthcare; IXICO Ltd.; Janssen Alzheimer Immunotherapy Research \& Development, LLC; Johnson \& Johnson Pharmaceutical Research \& Development LLC; Lumosity; Lundbeck; Merck \& Co., Inc; Meso Scale Diagnostics, LLC; NeuroRx Research; Neurotrack Technologies; Novartis Pharmaceuticals Corporation; Pfizer Inc:; Piramal Imaging; Servier; Takeda Pharmaceutical Company; and Transition Therapeutics. The Canadian Institutes of Health Research is providing funds to support ADNI clinical sites in Canada. Private sector contributions are facilitated by the Foundation for the National Institutes of Health (https://fnih.org/). The grantee organization is the Northern California Institute for Research and Education, and the study is coordinated by the Alzheimer's Disease Cooperative Study at the University of California, San Diego. ADNI data are disseminated by the Laboratory of Neuro Imaging at the University of Southern California.

\section{Availability of data and materials}

Data used in the preparation of this article were obtained from the Alzheimer's Disease Neuroimaging Initiative (ADNI) database (adni.loni.usc.edu).

\section{Authors' contributions}

$\mathrm{JKH}$ and DAN were responsible for the study concept and design, analysis and interpretation of data, statistical analysis, and preparation and editing of manuscript. Both authors read and approved the final manuscript.

\section{Competing interests}

The authors declare that they have no competing interests.

\section{Consent for publication}

Not applicable.

Ethics approval and consent to participate

Not applicable.

\section{Publisher's Note}

Springer Nature remains neutral with regard to jurisdictional claims in published maps and institutional affiliations.

Received: 6 September 2016 Accepted: 24 March 2017

Published online: 26 April 2017

\section{References}

1. Veglio F, Paglieri C, Rabbia F, Bisbocci D, Bergui M, Cerrato P. Hypertension and cerebrovascular damage. Atherosclerosis. 2009;205:331-41.

2. Duron E, Hanon O. Hypertension, cognitive decline and dementia. Arch Cardiovasc Dis. 2008;101:181-9.

3. de la Torre JC. Alzheimer disease as a vascular disorder: nosological evidence Stroke. 2002:33:1152-62.

4. Zlokovic BV. Neurovascular pathways to neurodegeneration in Alzheimer's disease and other disorders. Nat Rev Neurosci. 2011;12:723-38.

5. Skoog I, Gustafson D. Update on hypertension and Alzheimer's disease. Neurol Res. 2006;28:605-11.

6. Novak V, Haijar I. The relationship between blood pressure and cognitive function. Nat Rev Cardiol. 2010;7:686-98.

7. Christensen $\mathrm{H}$. What cognitive changes can be expected with normal ageing? Aust N Z J Psychiatry. 2001;35:768-75.

8. Knopman D, Boland LL, Mosley T, Howard G, Liao D, Szklo M, et al. Cardiovascular risk factors and cognitive decline in middle-aged adults. Neurology. 2001:56:42-8.

9. Kivipelto M, Helkala EL, Hanninen T, Laakso MP, Hallikainen M, Alhainen K, et al. Midlife vascular risk factors and late-life mild cognitive impairment: a population-based study. Neurology. 2001;56:1683-9.

10. Waldstein SR, Rice SC, Thayer JF, Najjar SS, Scuteri A, Zonderman AB. Pulse pressure and pulse wave velocity are related to cognitive decline in the Baltimore Longitudinal Study of Aging. Hypertension. 2008;51:99-104.

11. Saxby BK, Harrington F, McKeith IG, Wesnes K, Ford GA. Effects of hypertension on attention, memory, and executive function in older adults. Health Psychol. 2003;22:587-91.

12. Waldstein SR. The relation of hypertension to cognitive function. Curr Dir Psychol Sci. 2003;12:9-12.

13. Nation D, Wierenga C, Delano-Wood L, Jak A, Delis D, Salmon D, et al. Elevated pulse pressure is associated with age-related decline in language ability. J Int Neuropsychol Soc. 2010;16:933-8.

14. ladecola C, Davisson RL. Hypertension and cerebrovascular dysfunction. Cell Metab. 2008:7:476-84.

15. Gentile MT, Poulet R, Di Pardo A, Cifelli G, Maffei A, Vecchione C, et al. $\beta$-Amyloid deposition in brain is enhanced in mouse models of arterial hypertension. Neurobiol Aging. 2009;30:222-8. 
16. Langbaum JB, Chen K, Launer $\sqcup$, Fleisher AS, Lee W, Liu X, et al. Blood pressure is associated with higher brain amyloid burden and lower glucose metabolism in healthy late middle-age persons. Neurobiol Aging. 2012;33:827.e11-9.

17. Rodrigue KM, Rieck JR, Kennedy KM, Devous MD, Diaz-Arrastia R, Park DC. Risk factors for $\beta$-amyloid deposition in healthy aging: vascular and genetic effects. JAMA Neurol. 2013;70:600-6.

18. Nation DA, Edland SD, Bondi MW, Salmon DP, Delano-Wood L, Peskind ER, et al. Pulse pressure is associated with Alzheimer biomarkers in cognitively normal older adults. Neurology. 2013;81:2024-7.

19. Qiu C, Winblad B, Viitanen M, Fratiglioni L. Pulse pressure and risk of Alzheimer disease in persons aged 75 years and older: a community-based, longitudinal study. Stroke. 2003;34:594-9.

20. Fournier A, Oprisiu-Fournier R, Serot J, Godefroy O, Achard J, Faure S, et al. Prevention of dementia by antihypertensive drugs: how AT1-receptor-blockers and dihydropyridines better prevent dementia in hypertensive patients than thiazides and ACE-inhibitors. Expert Rev Neurother. 2009;9:1413-31.

21. McGuinness B, Todd S, Passmore P, Bullock R. Blood pressure lowering in patients without prior cerebrovascular disease for prevention of cognitive impairment and dementia. Cochrane Database Syst Rev. 2009;4, CD004034.

22. Prince MJ, Bird AS, Blizard RA, Mann AH. Is the cognitive function of older patients affected by antihypertensive treatment? Results from 54 months of the Medical Research Council's trial of hypertension in older adults. BMJ. 1996;312:801-5.

23. Tzourio C, Anderson C, Chapman N, Woodward M, Neal B, MacMahon S, et al. Effects of blood pressure lowering with perindopril and indapamide therapy on dementia and cognitive decline in patients with cerebrovascular disease. Arch Intern Med. 2003;163:1069-75.

24. Applegate WB, Pressel S, Wittes J, Luhr J, Shekelle RB, Camel GH, et al. Impact of the treatment of isolated systolic hypertension on behavioral variables: results from the Systolic Hypertension in the Elderly Program. Arch Intern Med. 1994;154:2154-60.

25. Di Bari M, Pahor M, Franse LV, Shorr RI, Wan JY, Ferrucci L, et al. Dementia and disability outcomes in large hypertension trials: lessons learned from the systolic hypertension in the elderly program (SHEP) trial. Am J Epidemiol. 2001;153:72-8.

26. Hoffman LB, Schmeidler J, Lesser GT, Beeri MS, Purohit DP, Grossman HT, et al. Less Alzheimer disease neuropathology in medicated hypertensive than nonhypertensive persons. Neurology. 2009;72:1720-6.

27. Haag MD, Hofman A, Koudstaal PJ, Breteler MM, Stricker BH. Duration of antihypertensive drug use and risk of dementia: a prospective cohort study. Neurology. 2009;72:1727-34.

28. Levi Marpillat N, Macquin-Mavier I, Tropeano Al, Bachoud-Levi AC, Maison P. Antihypertensive classes, cognitive decline and incidence of dementia: a network meta-analysis. J Hypertens. 2013;31:1073-82.

29. Fogari R, Mugellini A, Zoppi A, Derosa G, Pasotti C, Fogari E, et al. Influence of losartan and atenolol on memory function in very elderly hypertensive patients. J Hum Hypertens. 2003;17:781-5.

30. Hajjar I, Hart M, Chen Y, Mack W, Novak V, Chui CH, et al. Antihypertensive therapy and cerebral hemodynamics in executive mild cognitive impairment: results of a pilot randomized clinical trial. J Am Geriatr Soc. 2013;61:194-201.

31. Petersen RC, Aisen PS, Beckett LA, Donohue MC, Gamst AC, Harvey DJ, et al, Alzheimer's Disease Neuroimaging Initiative (ADNI): clinical characterization. Neurology. 2010;74:201-9.

32. Furiya $Y$, Ryo M, Kawahara M, Kiriyama T, Morikawa M, Ueno S. Renin-angiotensin system blockers affect cognitive decline and serum adipocytokines in Alzheimer's disease. Alzheimers Dement. 2013;9:512-8.

33. Sink KM, Leng X, Williamson J, Kritchevsky SB, Yaffe K, Kuller L, et al. Angiotensin-converting enzyme inhibitors and cognitive decline in older adults with hypertension: results from the Cardiovascular Health Study. Arch Intern Med. 2009;169:1195-202.

34. Wolf PA, D'Agostino RB, Belanger AJ, Kannel WB. Probability of stroke: a risk profile from the Framingham Study. Stroke. 1991;22:312-8.

35. Wilson PW, D'Agostino RB, Levy D, Belanger AM, Silbershatz H, Kannel WB. Prediction of coronary heart disease using risk factor categories. Circulation. 1998;97:1837-47.

36. Jack Jr CR, Bernstein MA, Fox NC, Thompson P, Alexander G, Harvey D, et al. The Alzheimer's disease neuroimaging initiative (ADNI): MRI methods. J Magn Reson Imaging. 2008;27:685-91.

37. Christensen GE, Joshi SC, Miller MI. Volumetric transformation of brain anatomy. IEEE Trans Med Imaging. 1997:16:864-77.
38. Schwarz C, Fletcher E, DeCarli C, Carmichael O. Fully-automated white matter hyperintensity detection with anatomical prior knowledge and without FLAIR. Inform Process Med Imaging. 2009;21:239-51.

39. Carmichael O, Mungas D, Beckett L, Harvey D, Farias ST, Reed B, et al. MR predictors of cognitive change in a diverse and carefully characterized elderly population. Neurobiol Aging. 2012;33:83-95.e2.

40. Strachan MW, Reynolds RM, Marioni RE, Price JF. Cognitive function, dementia and type 2 diabetes mellitus in the elderly. Nat Rev Endocrinol. 2011;7:108-14.

41. Li NC, Lee A, Whitmer RA, Kivipelto M, Lawler E, Kazis LE, et al. Use of angiotensin receptor blockers and risk of dementia in a predominantly male population: prospective cohort analysis. BMJ. 2010;340:b5465.

42. Davies NM, Kehoe PG, Ben-Shlomo Y, Martin RM. Associations of antihypertensive treatments with Alzheimer's disease, vascular dementia, and other dementias. J Alzheimers Dis. 2011:26:699-708.

43. Kehoe PG, Miners S, Love S. Angiotensins in Alzheimer's disease - friend or foe? Trends Neurosci. 2009:32:619-28.

44. Anderson C, Teo K, Gao P, Arima H, Dans A, Unger T, et al. Renin-angiotensin system blockade and cognitive function in patients at high risk of cardiovascular disease: analysis of data from the ONTARGET and TRANSCEND studies. Lancet Neurol. 2011;10:43-53.

45. Ohrui T, Matsui T, Yamaya M, Arai H, Ebihara S, Maruyama M, et al. Angiotensin-converting enzyme inhibitors and incidence of Alzheimer's disease in Japan. J Am Geriatr Soc. 2004;52:649-50.

46. Ohrui T, Tomita N, Sato-Nakagawa T, Matsui T, Maruyama M, Niwa K, et al. Effects of brain-penetrating ACE inhibitors on Alzheimer disease progression. Neurology. 2004;63:1324-5.

47. Reed BR, Mungas DM, Kramer JH, Ellis W, Vinters HV, Zarow C, et al. Profiles of neuropsychological impairment in autopsy-defined Alzheimer's disease and cerebrovascular disease. Brain. 2007;130:731-9.

48. Ciobica A, Bild W, Hritcu L, Haulica I. Brain renin-angiotensin system in cognitive function: pre-clinical findings and implications for prevention and treatment of dementia. Acta Neurol Belg. 2009:109:171-80.

49. Llorens-Cortes C, Mendelsohn FA. Organisation and functional role of the brain angiotensin system. J Renin Angiotensin Aldosterone Syst. 2002;3 Suppl 1:S39-48.

50. Suzuki Y, Ruiz-Ortega M, Lorenzo O, Ruperez M, Esteban V, Egido J. Inflammation and angiotensin II. Int J Biochem Cell Biol. 2003;35:881-900.

51. Iwai M, Liu HW, Chen R, Ide A, Okamoto S, Hata R, et al. Possible inhibition of focal cerebral ischemia by angiotensin II type 2 receptor stimulation. Circulation. 2004;110:843-8.

52. Nation DA, Ho J, Yew B. Older adults taking AT1-receptor blockers exhibit reduced cerebral amyloid retention. J Alzheimers Dis. 2016;50:779-89.

53. Weber MA, Schiffrin EL, White WB, Mann S, Lindholm LH, Kenerson JG, et al. Clinical practice guidelines for the management of hypertension in the community. J Clin Hypertens. 2014;16:14-26.

\section{Submit your next manuscript to BioMed Central and we will help you at every step:}

- We accept pre-submission inquiries

- Our selector tool helps you to find the most relevant journal

- We provide round the clock customer support

- Convenient online submission

- Thorough peer review

- Inclusion in PubMed and all major indexing services

- Maximum visibility for your research

Submit your manuscript at www.biomedcentral.com/submit 